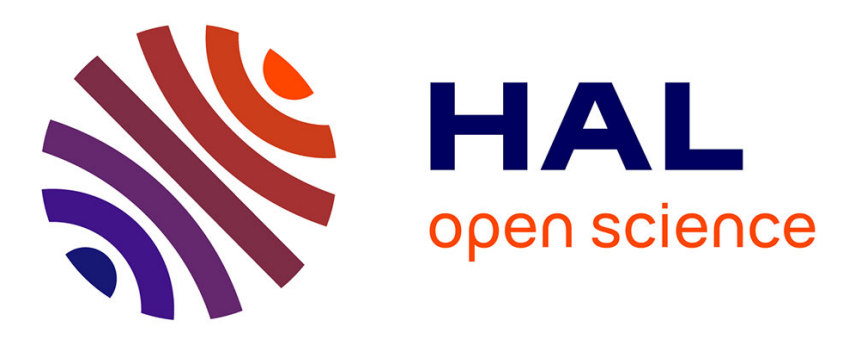

\title{
Optimal Control of Urban Human Mobility for Epidemic Mitigation
}

\author{
Muhammad Umar B. Niazi, Carlos Canudas de Wit, Alain Kibangou, \\ Pierre-Alexandre Bliman
}

\section{- To cite this version:}

Muhammad Umar B. Niazi, Carlos Canudas de Wit, Alain Kibangou, Pierre-Alexandre Bliman. Optimal Control of Urban Human Mobility for Epidemic Mitigation. CDC 2021 - 60th IEEE Conference on Decision and Control, Dec 2021, Austin, United States. hal-03185149

\section{HAL Id: hal-03185149 \\ https://hal.science/hal-03185149}

Submitted on 30 Mar 2021

HAL is a multi-disciplinary open access archive for the deposit and dissemination of scientific research documents, whether they are published or not. The documents may come from teaching and research institutions in France or abroad, or from public or private research centers.
L'archive ouverte pluridisciplinaire HAL, est destinée au dépôt et à la diffusion de documents scientifiques de niveau recherche, publiés ou non, émanant des établissements d'enseignement et de recherche français ou étrangers, des laboratoires publics ou privés. 


\title{
Optimal Control of Urban Human Mobility for Epidemic Mitigation
}

\author{
Muhammad Umar B. Niazi
}

Carlos Canudas-de-Wit

Alain Kibangou

Pierre-Alexandre Bliman

\begin{abstract}
Controlling human mobility during an epidemic is a fundamental issue faced by policymakers. Such control can only be done optimally if human mobility is adequately modeled at the scale of a city or metropolis. This paper, first, develops a model of human mobility that captures the daily patterns of mobility in an urban environment through time-dependent gating functions, which are controlled by the destination schedules and mobility windows. The process of epidemic spread is incorporated at each location that depends on the number of susceptible and infected people present at that location. Then, two optimal control policies are proposed to maximize the economic activity at the destinations while mitigating the epidemic. Precisely, operating capacities and time schedules of destinations are controlled to maximize the economic activity under the constraint that the number of active infected cases remains bounded.
\end{abstract}

\section{INTRODUCTION}

Although human mobility plays a vital role in a country's economy, it facilitates the spread of disease in the event of an epidemic by allowing contact between infected and susceptible populations. If human mobility is not controlled during an epidemic, it can result in a huge number of infected cases, which could overwhelm the hospitals and cause the loss of lives. On the other hand, strict restrictions on human mobility can also halt the economy and result in the loss of livelihoods. Therefore, the models of human mobility that incorporate the process of disease spread play a vital role in the analysis, understanding, and mitigation of epidemics.

Considering a SIR epidemic model for disease spread, human mobility between different geographic regions has been investigated and modeled in [1]-[4]. In these models, the individuals associated with one region or city can go to and return from other regions. However, these models capture averaged mobility patterns between different cities with large timescales and cannot capture the daily patterns of mobility within an urban environment. To tackle this problem, [5]-[8] study agent-based models of urban human mobility with epidemic spread. These models are powerful tools for computational purposes, however, they rely on the digital footprints of individuals and can lead to privacy violations. Another line of research in [9] relates to the

M.U.B. Niazi, C. Canudas-de-Wit, and A. Kibangou are with Université Grenoble Alpes, Inria, CNRS, Grenoble INP, GIPSA-Lab, 38000 Grenoble, France. Emails: muhammad-umar-b.niazi@gipsa-lab.fr carlos.canudas-dewit@gipsa-lab.fr alain.kibangou@univ-grenoble-alpes.fr

P.-A. Bliman is with Sorbonne Université, Université Paris-Diderot SPC, Inria, CNRS, Laboratoire Jacques-Louis Lions, équipe Mamba, 75005 Paris, France. Email: pierre-alexandre.bliman@inria.fr

This work is partially supported by European Research Council (ERC) under the European Union's Horizon 2020 research and innovation programme (ERCAdG no. 694209, Scale-FreeBack, website: http:// scale-freeback.eu/) and by Inria, France, in the framework of the Inria Mission Covid-19 (Project HealthyMobility). control of human mobility. It aims to find control policies that restrict mobility to and from regions that are estimated to be of high risk by employing reinforcement learning framework and relying on aggregated demand for mobility and regional epidemic statistics.

In this paper, we develop an urban human mobility model that captures the daily mobility patterns and incorporates the process of epidemic spread at each location. Every day a certain number of people go from their residential areas, which are called origins, to locations visited daily for work, education, shopping, etc., which are called destinations, and return on the same day. The daily mobility patterns are captured by the time-dependent supply and demand gating functions. The supply gating function (SGF) of each destination is controlled by its daily destination schedule, which is its opening and closing hours. The demand gating function (DGF), on the other hand, is defined on each edge of the mobility network and corresponds to the daily mobility window, which is the time interval during which people utilize that edge to move between origins and destinations. The supply function of each destination controlled by the SGF determines the inflow allowed to that destination and depends on its operating capacity controlled by the capacity control input. The demand function controlled by the DGF determines the outflow from one location to another. The process of urban human mobility is modeled on the network edges that connect different locations through flows and the process of epidemic spread is modeled locally at each location that depends on the number of susceptible and infected people at that location.

We formulate two optimal control problems for epidemic mitigation while maximizing the economic activity: (i) optimal capacity control policy and (ii) optimal schedule control policy. These problems aim to find an optimal capacity control input and schedule control input, respectively, that maximizes the economic activity while mitigating the epidemic by keeping the number of active infected cases bounded. The capacity control policy restrict the number of people in destinations of each category by specifying the operating capacities in relation to their nominal capacities. The schedule control inputs, on the other hand, specifies the closing hour of destinations of each category by altering the destination schedules and mobility windows.

The paper is organized as follows: Section $[\mathrm{II}$ introduces the urban human mobility model that incorporates the process of epidemic spread at each location. Section III presents optimal capacity control and optimal schedule control policies, and illustrates their efficacy through a numerical example. Finally, Section IV presents the concluding remarks. 


\section{Model of URban Human Mobility with EPIDEMIC SPREAD}

Consider human mobility in an urban environment between locations of two types: origins and destinations. The origins correspond to locations where people reside-for example, residential areas, neighborhoods, and towns. The destinations, on the other hand, correspond to locations that people visit daily for work, education, shopping, or leisurefor example, industrial zones, business parks, schools, markets, cinemas, etc. Every day a certain number of people go from each origin to the destinations during specified time intervals and then return later the same day. At each location, there is an epidemic spread process that will be described here by a SIR model, [10], where the population is divided into Susceptible, Infected, and Recovered classes, and the disease is transmitted according to the local infection rates when the susceptible and infected populations mix in the same location. Notice that any similar epidemiological model could be used instead.

\section{A. Network Representation and Main Assumptions}

Let $\mathcal{V}_{o}=\{1, \ldots, m\}$ be the index set of $m$ origins and $\mathcal{V}_{d}=\{m+1, \ldots, m+n\}$ be the index set of $n$ destinations. The network of urban human mobility is represented by a bi-directed, bipartite graph $\mathcal{G}=\left(\mathcal{V}_{o}, \mathcal{V}_{d}, \mathcal{E}\right)$, where $\mathcal{E}$ is the set of bi-directed edges-i.e., for every $i \in \mathcal{V}_{o}$ and $j \in \mathcal{V}_{d}$, if $(i, j) \in \mathcal{E}$ then $(j, i) \in \mathcal{E}$. Figure 1 illustrates a mobility network, which is the running example of this paper.

We adopt the following assumptions in the model:

(A1) The total population of the city remains constant.

(A2) The mobility occurs only between pairs of origins and destinations, and not among a pair of different origins or a pair of different destinations.

(A3) The number of people who visit destination $j$ from origin $i$ during a day is equal to the number of people who return to $i$ from $j$ on the same day.

(A4) The mobility pattern between each pair of origins and destinations is periodic and repeats every day, i.e., the period $T_{\text {period }}=24$ hours. In particular, we ignore mobility patterns of the weekends or holidays that may be different than the normal days.

(A5) The restrictions imposed on the urban human mobility by the government affects all the people, whether susceptible, infected, or recovered, equally.

Note that (A4) is a simplifying assumption, which can be relaxed without loss of generality by considering $T_{\text {period }}$ to be a week, a month, etc.

Denote $P_{i}$ to be the total population of origin $i \in \mathcal{V}_{o}$, which is the number of people who reside in $i$, and $C_{j}$ to be the nominal capacity of destination $j \in \mathcal{V}_{d}$, which is the maximum number of people who can visit $j$ at one time when there is no epidemic. Note that the total population of the city $P=\sum_{i \in \mathcal{V}_{o}} P_{i}$.

\section{B. Destination Categories}

Suppose the destinations are divided into $p \leq n$ categories, which correspond to workplace, school, market, etc. The

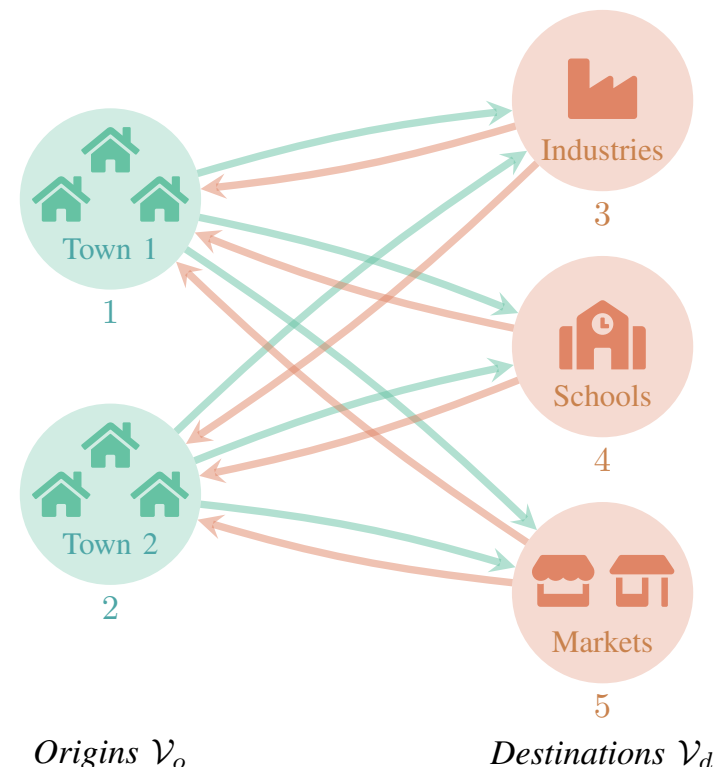

Fig. 1: An example of an urban human mobility network with two origins and three destinations.

categories of destinations are represented by a partition

$$
\mathcal{D}=\left\{\mathcal{D}_{1}, \ldots, \mathcal{D}_{p}\right\}
$$

where the destinations of category $k$ are grouped in a set $\mathcal{D}_{k}$, for $k=1, \ldots, p$, and each destination belongs to only one category, i.e., for every $k, l \in\{1, \ldots, p\}$ and $l \neq k$,

$$
\bigcup_{k=1}^{p} \mathcal{D}_{k}=\mathcal{V}_{d} \quad \text { and } \quad \mathcal{D}_{k} \cap \mathcal{D}_{l}=\emptyset
$$

In the example illustrated in Figure 11, we have $p=n$, i.e., three categories and three destinations, because each destination is of a different category. However, it is possible that a mobility network may have multiple destinations of the same category. For instance, a slight modification of the example of Figure 1, say with two working places and three schools, could illustrate this immediately.

\section{Operating Capacities of Destinations}

Let $u_{k}(t) \in[0,1]$ be the capacity control input for destinations of category $k$, for $k=1, \ldots, p$, which determines the allowed operating capacity of $\mathcal{D}_{k}$ in terms of the proportion of nominal capacity at time $t$ in the event of an epidemic. In other words, it can be considered as the government's policy at time $t$ that limits the operating capacities in destinations of category $k$ in order to mitigate the epidemic spread, where

$$
\text { Operating capacity }=C_{j} u_{k}(t), \quad \text { for } j \in \mathcal{D}_{k} \text {. }
$$

We consider $u_{k}(t)$ to be piece-wise constant-i.e.,

$$
u_{k}(t)=\left\{\begin{array}{cl}
\mu_{k}^{1} & \text { if } t \in\left[0, T_{u}\right) \\
\mu_{k}^{2} & \text { if } t \in\left[T_{u}, 2 T_{u}\right) \\
\vdots & \vdots \\
\mu_{k}^{q} & \text { if } t \in\left[(q-1) T_{u}, q T_{u}\right)
\end{array}\right.
$$


with $\mu_{k}^{h} \in[0,1]$ constant for every $h \in\{1, \ldots, q\}$ and $T=$ $q T_{u}$ the total time horizon considered by a policymaker. The policy horizon $T_{u}$ is a multiple of $T_{\text {period }}$ and corresponds to the time after which the policy on operating capacities is announced periodically. It can be chosen by the policymaker from at least a week to several months because changing the policy on shorter time intervals may not be practical in terms of implementation.

\section{Destination Schedule and Mobility Window}

The destination schedule of $j \in \mathcal{V}_{d}$

$$
\mathbb{S}_{j}=\left[a_{j}, b_{j}\right), \quad 0 \leq a_{j}<b_{j} \leq 24
$$

is the daily time interval during which $j$ is open, where $a_{j}$ and $b_{j}$ are the nominal opening and closing hours of $j$, respectively. The origins, on the other hand, are open throughout the day, i.e., for every $i \in \mathcal{V}_{o}, \mathbb{S}_{i}=[0,24)$. Then, the supply gating function (SGF) for $j \in \mathcal{V}_{o} \cup \mathcal{V}_{d}$

$$
\sigma_{j}(t)= \begin{cases}1 & \text { if } t \bmod 24 \in \mathbb{S}_{j} \\ 0 & \text { otherwise }\end{cases}
$$

which is periodic with respect to 24 hours.

The mobility window of $(i, j) \in \mathcal{E}$

$$
\mathbb{D}_{i j}=\left[t_{i j}, t_{i j}+\tau_{i j}\right), \quad 0 \leq t_{i j}<t_{i j}+\tau_{i j} \leq 24
$$

is the daily time interval during which there is mobility from $i$ to $j$, where $\tau_{i j}>0$ is the duration of mobility window in hours. Then, the demand gating function (DGF) of $(i, j) \in \mathcal{E}$

$$
\delta_{i j}(t)= \begin{cases}1 & \text { if } t \bmod 24 \in \mathbb{D}_{i j} \\ 0 & \text { otherwise }\end{cases}
$$

which is also periodic with respect to 24 hours.

\section{E. Model of Urban Human Mobility}

Let $N_{i}(t) \geq 0$ be the number of people in $i \in \mathcal{V}_{o} \cup \mathcal{V}_{d}$ at time $t$ (hour). Then, according to the urban human mobility model, the rate of change of the number of people at any location at time $t$ is equal to the sum of inflows to that location minus the sum of outflows from that location.

In other words, for any $i \in \mathcal{V}_{o} \cup \mathcal{D}_{k}$ and $k \in\{1, \ldots, p\}$, the urban human mobility model is given by

$$
\dot{N}_{i}=\sum_{j \in \mathcal{N}_{i}}\left(\phi_{j i}-\phi_{i j}\right)
$$

where $\mathcal{N}_{i}$ is the set of neighbors of $i$ in the mobility network $\mathcal{G}$ and $\phi_{i j}\left(t, N_{i}(t), N_{j}(t), u_{k}(t)\right)$ is the flow from $i \in \mathcal{V}_{o}$ to $j \in \mathcal{D}_{k}$ given as

$$
\phi_{i j}=\min \left(\Delta_{i j}, \Sigma_{j}\right)
$$

with $\Delta_{i j}\left(t, N_{i}(t), u_{k}(t)\right)$ and $\Sigma_{j}\left(t, N_{j}(t), u_{k}(t)\right)$ the demand and supply functions, respectively. Notice that the flow $\phi_{j i}\left(t, N_{j}(t), N_{i}(t), u_{k}(t)\right)$ is defined similarly with the subscript $j i$ instead of $i j$.

The supply function $\Sigma_{j}\left(t, N_{j}(t), u_{k}(t)\right)$ of each location $j$ corresponds to the allowed inflow to $j$ from other locations and is given by

$$
\Sigma_{j}= \begin{cases}\sigma_{j} \min \left(F_{j}, v\left[C_{j} u_{k}-N_{j}\right]\right) & \text { if } j \in \mathcal{D}_{k} \\ \sigma_{j} \min \left(F_{j}, v\left[P_{j}-N_{j}\right]\right) & \text { if } j \in \mathcal{V}_{o}\end{cases}
$$
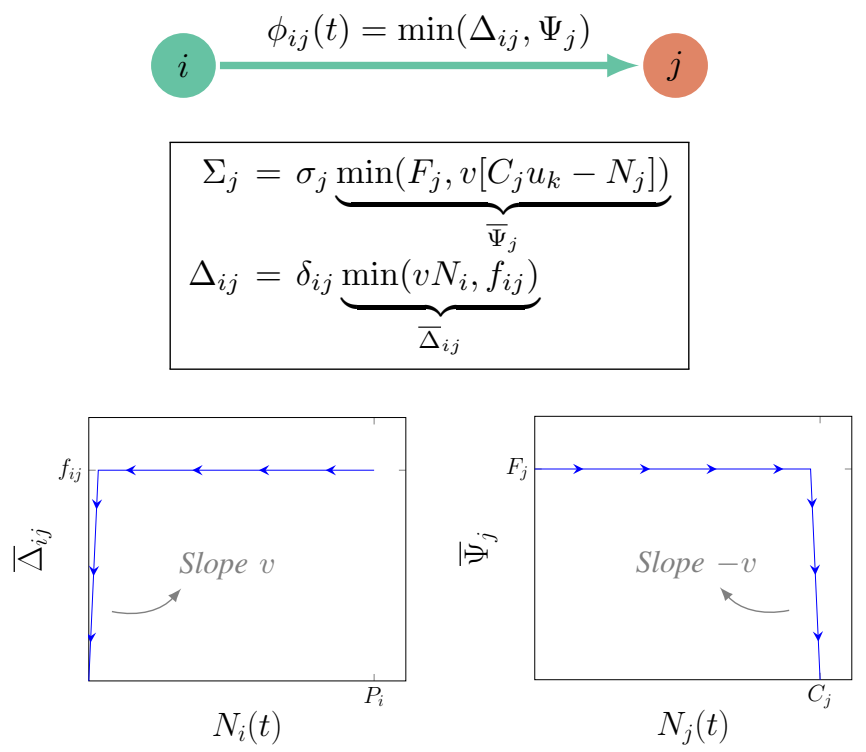

Fig. 2: An example illustrating the flow $\phi_{i j}(t)$ from origin $i$ to destination $j$ in terms of supply of $j$ and demand of $i$ with respect to $j$. Here, the arrows on each curve indicate the time evolution.

where $\sigma_{j}(t)$ is the SGF given by (2), $v>0$ is a regularization parameter taken to be very large (see Remark $1, C_{j} u_{k}(t)$ is the operating capacity of $j \in \mathcal{D}_{k}$ with $u_{k}(t)$ defined in (1), and

$$
F_{j}(t)=\sum_{i \in \mathcal{N}_{j}} f_{i j}(t)
$$

is the maximum inflow to $j$ with

$$
f_{i j}(t)=\frac{M_{i j} u_{k}(t)}{\tau_{i j}}
$$

the maximum outflow from $i \in \mathcal{V}_{o}$ to $j \in \mathcal{D}_{k}$. Here, $M_{i j}$ denotes the nominal number of visitors to $j$ from $i$ and $M_{i j} u_{k}(t)$ is the number of visitors when the capacity control input $u_{k}(t)$ is implemented. Notice that, since all visitors return, we have $M_{j i}=M_{i j}$.

The demand function $\Delta_{i j}\left(t, N_{i}(t), u_{k}(t)\right)$ of each edge $(i, j) \in \mathcal{E}$ corresponds to the outflow from $i$ towards $j$ and is given by

$$
\Delta_{i j}=\delta_{i j} \min \left(v N_{i}, f_{i j}\right)
$$

where $\delta_{i j}(t)$ is the DGF given by (3), $v>0$ is the same regularization parameter introduced in the supply function $\Sigma_{j}\left(t, N_{j}(t), u_{k}(t)\right)$, and $f_{i j}(t)$ is the maximum outflow from $i$ to $j$ given by (5).

Suppose $i \in \mathcal{V}_{o}$ and $j \in \mathcal{D}_{k}$, then Figure 2 illustrates the flow from $i$ to $j$ in terms of demand and supply functions. In the figure, notice that the demand of $i$ moves from right to left with respect to time $t$, i.e., from being full to being empty, and the supply of $j$ moves from left to right with respect to time $t$, i.e., from being empty to being full, which is indicated by arrows in the figure. 
Remark 1. To ensure that the daily number of people going from $i$ to $j$ equals $M_{i j} u_{k}(t)$, we assume that the demand function $\Delta_{i j}\left(t, N_{i}(t), u_{k}(t)\right)=\delta_{i j}(t) f_{i j}(t) \mathbb{1}_{N_{i}(t)>0}$, where $\mathbb{1}_{N_{i}(t)>0}=1$ if $N_{i}(t)>0$, and 0 otherwise, is the indicator function. Similarly, we assume that the supply function $\Psi_{j}\left(t, N_{j}(t), u_{k}(t)\right)=\sigma_{j}(t) F_{j}(t) \mathbb{1}_{N_{j}(t)<C_{j} u_{k}(t)}$. However, to avoid the discontinuity posed by the indicator functions, we approximate the demand and supply functions by considering steep slope with a very large regularization parameter $v$ as illustrated in Figure 2.

\begin{tabular}{|c|c|c|c|}
\hline Populations & $P_{1}=3000$ & $P_{2}=2000$ & \\
\hline Capacities & $C_{3}=2000$, & $C_{4}=1500$ & $C_{5}=200$ \\
\hline No. of Visitors & $\begin{array}{l}M_{13}=1200 \\
M_{23}=800\end{array}$ & $\begin{array}{l}M_{14}=900 \\
M_{24}=600\end{array}$ & $\begin{array}{l}M_{15}=900 \\
M_{25}=600\end{array}$ \\
\hline $\begin{array}{l}\text { Mobility } \\
\text { windows }\end{array}$ & $\begin{array}{l}\mathbb{D}_{i 3}=[8,9.5), \\
\mathbb{D}_{3 i}=[17,18.5),\end{array}$ & $\begin{array}{l}\mathbb{D}_{i 4}=[9,10), \\
\mathbb{D}_{4 i}=[16,17), \\
\text { for } i=1,2\end{array}$ & $\begin{aligned} \mathbb{D}_{i 5} & =[8.5,20) \\
\mathbb{D}_{5 i} & =[10,21)\end{aligned}$ \\
\hline $\begin{array}{l}\text { Destination } \\
\text { schedules }\end{array}$ & $\mathbb{S}_{3}=[8,18)$ & $\mathbb{S}_{4}=[9,17)$ & $\mathbb{S}_{5}=[8.5,20)$ \\
\hline $\begin{array}{l}\text { Capacity control } \\
\text { policy }\end{array}$ & $u_{1}(t)=0.5$ & $\begin{array}{l}u_{2}(t)=0.5 \\
\text { for } t \in[0,48)\end{array}$ & $u_{3}(t)=1$ \\
\hline $\begin{array}{l}\text { Regularization } \\
\text { parameter }\end{array}$ & $v=30$ & & \\
\hline
\end{tabular}

TABLE I: Parameters related to urban human mobility for the example of Figure 1

Example 1. Consider the example of mobility network shown in Figure 1 with two origins and three destinations. For the mobility model (4), we consider the parameters given in Table II The mobility profile of two days is plotted in Figure 3, where $N_{1}(t), N_{2}(t)$ denote the number of people in Town 1 and 2 at time $t$, respectively, and $N_{3}(t), N_{4}(t), N_{5}(t)$ denote the number of people in the industries, schools, and markets, respectively. As shown in the figure, people go from the origins ( 1 and 2) to the destinations (3, 4, and 5) and return on the same day according to the destination schedules and mobility windows. Notice that the mobility profiles are the same for both days because, by assumption (A4), the destination schedules and mobility windows are the same for every day. Moreover, the nominal capacities of industries and schools are $C_{3}=2000$ and $C_{4}=1500$, however, the capacity control policy $u_{1}(t)=u_{2}(t)=0.5$ reduce the operating capacities to $50 \%$ of the nominal capacities. Therefore, the maximum number of people present in these destinations during a day is around $C_{3} / 2=1000$ and $C_{4} / 2=750$, respectively.

\section{F. Incorporating the Epidemic Spread Process}

We consider a susceptible, infected, recovered (SIR) model of epidemic spread, which divides the number of people $N_{i}(t)$ at each location $i \in \mathcal{V}_{o} \cup \mathcal{V}_{d}$ into three classes: number of susceptible $S_{i}(t)$, infected $I_{i}(t)$, and recovered $R_{i}(t)$, where, at every time $t$,

$$
N_{i}(t)=S_{i}(t)+I_{i}(t)+R_{i}(t) .
$$

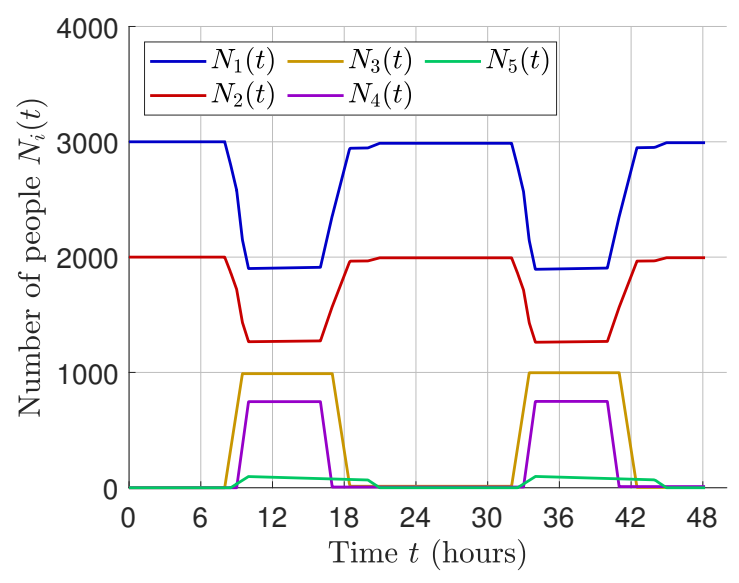

Fig. 3: The mobility profile of two days for the example in Figure 1 .

The disease transmission at each location $i$ occurs according to the local mass action law

$$
\beta_{i}(t) S_{i}(t) \frac{I_{i}(t)}{N_{i}(t)}
$$

where

$$
\beta_{i}(t)= \begin{cases}\bar{\beta}_{i} \frac{N_{i}(t)}{P_{i}} & \text { if } i \in \mathcal{V}_{o} \\ \bar{\beta}_{i} \frac{N_{i}(t)}{C_{i}} & \text { if } i \in \mathcal{V}_{d}\end{cases}
$$

is the infection rate of $i$ at time $t$ with $\bar{\beta}_{j}$ the nominal infection rate of $i$. The nominal infection rate is defined as the average number of contacts of a person in location $i$ per hour when the number of people in $i$ is maximum. The infection rate $\beta_{i}(t)$ reduces when the number of people $N_{i}(t)$ at location $i$ is small and increases when $N_{i}(t)$ is large. The infected people $I_{i}(t)$ recover with a recovery rate $\gamma \in(0,1]$, which is a constant that depends on the disease biology and, if available, the treatment methods. The recovery rate $\gamma$ is defined as the inverse of the average recovery period (in hours) of the infected cases.

Recall the assumption (A5), which implies that all people irrespective of whether they are susceptible, infected, or recovered can go from one location to another with equal probability. That is, the flow from $i$ to $j$ in terms of the number of susceptible, infected, and recovered can be respectively given by

$$
\begin{aligned}
& \phi_{i j}\left(t, N_{i}(t), N_{j}(t), u_{k}(t)\right) \frac{S_{i}(t)}{N_{i}(t)} \\
& \phi_{i j}\left(t, N_{i}(t), N_{j}(t), u_{k}(t)\right) \frac{I_{i}(t)}{N_{i}(t)} \\
& \phi_{i j}\left(t, N_{i}(t), N_{j}(t), u_{k}(t)\right) \frac{R_{i}(t)}{N_{i}(t)} .
\end{aligned}
$$

Let $\mathbf{x}_{i}(t)=\left[\begin{array}{lll}S_{i}(t) & I_{i}(t) & R_{i}(t)\end{array}\right]^{\mathrm{T}} \in \mathbb{R}_{\geq 0}^{3}$ be the state vector of location $i \in \mathcal{V}_{o} \cup \mathcal{V}_{d}$ and

$$
\boldsymbol{\xi}_{i}\left(\mathbf{x}_{i}(t)\right)=\left[\begin{array}{c}
-\beta_{i}(t) S_{i}(t) \frac{I_{i}(t)}{N_{i}(t)} \\
\beta_{i}(t) S_{i}(t) \frac{I_{i}(t)}{N_{i}(t)}-\gamma I_{i}(t) \\
\gamma I_{i}(t)
\end{array}\right] \in \mathbb{R}^{3}
$$




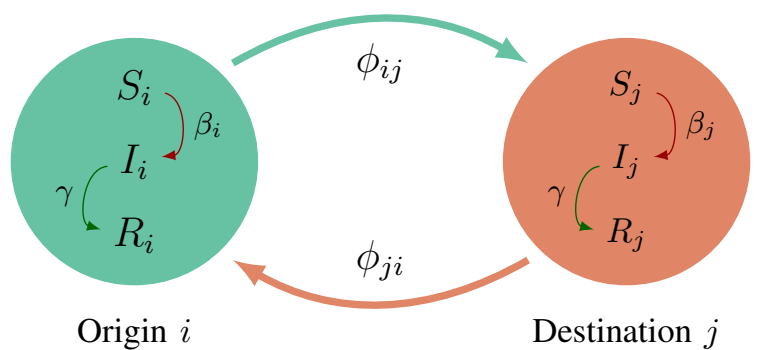

Fig. 4: The process of urban human mobility happens along the edges whereas the process of epidemic spread happens inside the locations.

be the vector describing the process of epidemic spread in location $i$. Then, for $i \in \mathcal{V}_{o}$ and $j \in \mathcal{D}_{k}$, the model of urban human mobility with epidemic spread is given by

$$
\begin{aligned}
& \dot{\mathbf{x}}_{i}=\boldsymbol{\xi}_{i}\left(\mathbf{x}_{i}\right)+\sum_{k=1}^{p} \sum_{j \in \mathcal{D}_{k}}\left[\phi_{j i}\left(u_{k}\right) \frac{\mathbf{x}_{j}}{N_{j}}-\phi_{i j}\left(u_{k}\right) \frac{\mathbf{x}_{i}}{N_{i}}\right] \\
& \dot{\mathbf{x}}_{j}=\boldsymbol{\xi}_{j}\left(\mathbf{x}_{j}\right)+\sum_{i \in \mathcal{V}_{o}}\left[\phi_{i j}\left(u_{k}\right) \frac{\mathbf{x}_{i}}{N_{i}}-\phi_{j i}\left(u_{k}\right) \frac{\mathbf{x}_{j}}{N_{j}}\right] .
\end{aligned}
$$

As illustrated in Figure 4, there are two aspects of the model. First, inside the locations $i$ and $j$, there is a process of epidemic spread that transmits the disease from the infected to the susceptible with a local infection rate, and the recovery process that heals the infected with a constant recovery rate. Second, on the edges $(i, j)$ and $(j, i)$, there is a process of human mobility that transfers people from one location to another through the flows $\phi_{i j}\left(t, N_{i}(t), N_{j}(t), u_{k}(t)\right)$ and $\phi_{j i}\left(t, N_{j}(t), N_{i}(t), u_{k}(t)\right)$, respectively.

\section{Optimal Mobility Control for EpIDEMIC Mitigation}

\section{A. Compact Representation of the Model}

The urban human mobility model with epidemic spread given in (7) describes the dynamics of the number of susceptible $S_{i}(t)$, infected $I_{i}(t)$, and recovered $R_{i}(t)$ people in location $i \in \mathcal{V}_{o} \cup \mathcal{V}_{d}$. These dynamics are controlled by the piece-wise constant capacity control inputs $u_{1}(t), \ldots, u_{p}(t)$, defined in (1), of the $p$ destination categories in $\mathcal{D}$. Define

$$
\mathbf{u}(t)=\left[\begin{array}{lll}
u_{1}(t) & \ldots & u_{p}(t)
\end{array}\right]^{\mathrm{T}} .
$$

Then, to represent the model in a compact form, let

$$
\mathbf{x}=\left[\begin{array}{l}
\mathbf{x}_{o} \\
\mathbf{x}_{d}
\end{array}\right], \quad \text { where }\left\{\begin{array}{l}
\mathbf{x}_{o}=\left[\begin{array}{lll}
\mathbf{x}_{1}^{\mathrm{T}} \cdots & \mathbf{x}_{m}^{\mathrm{T}}
\end{array}\right]^{\mathrm{T}} \\
\mathbf{x}_{d}=\left[\begin{array}{lll}
\mathbf{x}_{m+1}^{\mathrm{T}} & \cdots & \mathbf{x}_{m+n}^{\mathrm{T}}
\end{array}\right]^{\mathrm{T}}
\end{array}\right.
$$

with $\mathbf{x}_{o}(t) \in \mathbb{R}_{\geq 0}^{3 m}$ the state vector of origins $\mathcal{V}_{o}$ and $\mathbf{x}_{d}(t) \in$ $\mathbb{R}_{\geq 0}^{3 n}$ the state vector of destinations $\mathcal{V}_{d}$. Similarly, let

$$
\boldsymbol{\xi}=\left[\begin{array}{l}
\boldsymbol{\xi}_{o} \\
\boldsymbol{\xi}_{d}
\end{array}\right], \quad \text { where }\left\{\begin{array}{l}
\boldsymbol{\xi}_{o}=\left[\begin{array}{lll}
\boldsymbol{\xi}_{1}^{\mathrm{T}} & \cdots & \boldsymbol{\xi}_{m}^{\mathrm{T}}
\end{array}\right]^{\mathrm{T}} \\
\boldsymbol{\xi}_{d}=\left[\begin{array}{lll}
\boldsymbol{\xi}_{m+1}^{\mathrm{T}} & \cdots & \boldsymbol{\xi}_{m+n}^{\mathrm{T}}
\end{array}\right]^{\mathrm{T}}
\end{array}\right.
$$

with $\boldsymbol{\xi}_{o}\left(\mathbf{x}_{o}(t)\right) \in \mathbb{R}^{3 m}$ the vector describing the epidemic process in the origins $\mathcal{V}_{o}$ and $\boldsymbol{\xi}_{d}\left(\mathbf{x}_{d}(t)\right) \in \mathbb{R}^{3 n}$ the vector describing the epidemic process in the destinations $\mathcal{V}_{d}$ Notice that the vector $\boldsymbol{\xi}_{i}\left(\mathbf{x}_{i}(t)\right)$ describing the epidemic process in each location $i$ is given in (6).

The model (7) can be represented as

$$
\dot{\mathbf{x}}=\boldsymbol{\xi}(\mathbf{x})+\Phi(\mathbf{x}, \mathbf{u}) \mathbf{x}
$$

where the dependence on $t$ is omitted for brevity. The matrix of flows $\Phi(t, \mathbf{x}(t), \mathbf{u}(t))$ describes the mobility process in the network $\mathcal{G}$ and is given as

$$
\Phi(\mathbf{x}, \mathbf{u})=\left[\begin{array}{ll}
\Phi_{o o}(\mathbf{x}, \mathbf{u}) & \Phi_{d o}(\mathbf{x}, \mathbf{u}) \\
\Phi_{o d}(\mathbf{x}, \mathbf{u}) & \Phi_{d d}(\mathbf{x}, \mathbf{u})
\end{array}\right] \otimes I_{3}
$$

where $\otimes$ denotes the Kronecker product and

$$
\begin{aligned}
\Phi_{o o}= & \operatorname{diag}\left[-\sum_{j \in \mathcal{V}_{d}} \frac{\phi_{1 j}}{N_{1}} \ldots-\sum_{j \in \mathcal{V}_{d}} \frac{\phi_{m j}}{N_{m}}\right] \\
\Phi_{d d}= & \operatorname{diag}\left[-\sum_{i \in \mathcal{V}_{o}} \frac{\phi_{m+1, i}}{N_{m+1}} \ldots-\sum_{i \in \mathcal{V}_{o}} \frac{\phi_{m+n, i}}{N_{m+n}}\right] \\
\Phi_{o d}= & {\left[\begin{array}{ccc}
\frac{\phi_{1, m+1}}{N_{1}} & \cdots & \frac{\phi_{m, m+1}}{N_{m}} \\
\vdots & \ddots & \vdots \\
\frac{\phi_{1, m+n}}{N_{1}} & \cdots & \frac{\phi_{m, m+n}}{N_{m}}
\end{array}\right] } \\
\Phi_{d o}= & {\left[\begin{array}{ccc}
\frac{\phi_{m+1,1}}{N_{m+1}} & \cdots & \frac{\phi_{m+n, 1}}{N_{m+n}} \\
\vdots & \ddots & \vdots \\
\frac{\phi_{m+1, m}}{N_{m+1}} & \cdots & \frac{\phi_{m+n, m}}{N_{m+n}}
\end{array}\right] . }
\end{aligned}
$$

\section{B. Economic Activity and Active Infected Cases}

The economic activity $E(t) \in \mathbb{R}_{\geq 0}$ in the mobility network $\mathcal{G}$ at time $t$ is defined as

$$
E(t)=\sum_{k=1}^{p} \sum_{j \in \mathcal{D}_{k}} \chi_{j} \frac{N_{j}(t)}{C_{j}}
$$

where $N_{j}(t)$ is the number of people in destination $j \in \mathcal{D}_{k}$ at time $t, C_{j}$ is the nominal capacity of $j$, and $\chi_{j} \in[0,1]$ is the weight assigned to $j$ according to its economic importance such that $\sum_{j \in \mathcal{V}_{d}} \chi_{j}=1$. Since $N_{i}(t)=\mathbf{1}_{3}^{\mathrm{T}} \mathbf{x}_{i}(t)$, we can write (9) as

$$
E(t)=\mathbf{e}^{\mathrm{T}} \mathbf{x}(t)
$$

where $\mathbf{x}(t)$ is the state of $(8)$ at time $t$ and

$$
\mathbf{e}=\left[\begin{array}{llll}
\mathbf{0}_{m}^{\mathrm{T}} & \frac{\chi_{m+1}}{C_{m+1}} & \cdots & \frac{\chi_{m+n}}{C_{m+n}}
\end{array}\right]^{\mathrm{T}} \otimes\left[\begin{array}{lll}
1 & 1 & 1
\end{array}\right]^{\mathrm{T}} .
$$

The number of active infected cases $I(t) \in \mathbb{R}_{\geq 0}$ in the mobility network $\mathcal{G}$ at time $t$ is the sum of the number of infected people in all the locations. It is given by

$$
I(t)=\sum_{i \in \mathcal{V}_{o} \cup \mathcal{V}_{d}} I_{i}(t)
$$

where $I_{i}(t)$ is the number of infected people at location $i$ at time $t$. Since $I_{i}(t)=\left[\begin{array}{lll}0 & 1 & 0\end{array}\right] \mathbf{x}_{i}(t)$, we can write (11) as

$$
I(t)=\mathbf{g}^{\mathrm{T}} \mathbf{x}(t)
$$




\begin{tabular}{|l||l|}
\hline Recovery rate & $\gamma=1 / 14$ per 24 hours \\
\hline Nominal infection rates & $\bar{\beta}_{1}=0.11$ per 24 hours \\
& $\bar{\beta}_{2}=0.11$ per 24 hours \\
& $\bar{\beta}_{3}=0.71$ per 24 hours \\
& $\bar{\beta}_{4}=1.07$ per 24 hours \\
& $\bar{\beta}_{5}=0.57$ per 24 hours \\
\hline
\end{tabular}

TABLE II: Parameters related to local epidemic spread for the example of Figure 1

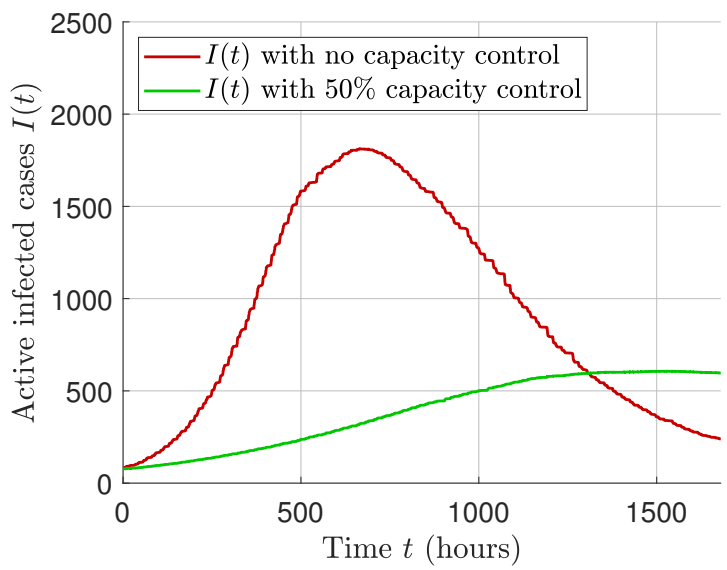

Fig. 5: Reduction of the number of infected cases $I(t)$ by reducing operating capacities via capacity control policy.

where

$$
\mathbf{g}=\left[\begin{array}{ll}
\mathbf{1}_{m}^{\mathrm{T}} & \mathbf{1}_{n}^{\mathrm{T}}
\end{array}\right]^{\mathrm{T}} \otimes\left[\begin{array}{lll}
0 & 1 & 0
\end{array}\right]^{\mathrm{T}} .
$$

Finally, the infection peak is defined as

$$
I_{\text {peak }}=\sup _{t \in[0, T]} I(t)=\sup _{t \in[0, T]} \mathbf{g}^{\mathrm{T}} \mathbf{x}(t)
$$

where $[0, T]$ is a given finite time horizon.

Example 2. Consider the example of mobility network in Figure 1 with the mobility parameters given in Table $\mathrm{I}$ and the epidemic parameters given in Table $\Pi$. Note that the nominal infection rates outside the residences (the "destinations") are assumed higher than the nominal infection rates at the residences (the "origins"). For $T=1680$ hours (or 10 weeks), we plot the active infected cases $I(t)$ in Figure 5 under two circumstances: (i) when there are no restrictions on the operating capacities of all destinations, i.e., $\mathbf{u}(t)=\left[\begin{array}{lll}1 & 1 & 1\end{array}\right]^{\mathrm{T}}$ for all $t \in[0, T]$, and (ii) when the operating capacities of all destinations are reduced to $50 \%$ throughout $[0, T]$, i.e., $\mathbf{u}(t)=\left[\begin{array}{lll}0.5 & 0.5 & 0.5\end{array}\right]^{\mathrm{T}}$ for all $t \in[0, T]$. In Figure 5 , notice the effect on the number of active infected cases when the operating capacities are reduced. When there are no restrictions on the operating capacities, the infection peak $I_{\text {peak }}$ is about 1810 people, whereas, with the restrictions, the peak is about 605 people. In other words, given the mobility and epidemic parameters for the example of Figure 1. one can reduce the infection peak by $66.5 \%$ through $50 \%$ reduction of the operating capacities.

\begin{tabular}{|l||l|}
\hline Time horizon & $T=1680$ hours $\quad(10$ weeks $)$ \\
\hline Policy horizon & $T_{u}=336$ hours $\quad(2$ weeks $)$ \\
\hline $\begin{array}{l}\text { Upper bound } \\
\text { on infection peak }\end{array}$ & $\bar{I}=1000$ \\
\hline $\begin{array}{l}\text { Weights of } \\
\text { economic importance }\end{array}$ & $\chi_{3}=0.4, \quad \chi_{4}=0.3, \quad \chi_{5}=0.3$ \\
\hline
\end{tabular}

TABLE III: Parameters related to the optimal control problem (13) for the example of Figure 1

\section{Optimal Capacity Control Policy}

The number of hospitalized cases and deaths due to an epidemic are related to the number of infected cases $I(t)$. A large value of $I(t)$ implies a large number of hospitalizations and loss of lives in the near future. Moreover, if the measures to mitigate the epidemic and limit the number of infected cases are not taken, then the number of hospitalizations may reach a point that could challenge the available medical facilities of the city. As shown in Example 2, the number of infected cases can be reduced by reducing the operating capacities of the destinations through the capacity control policies $u_{1}(t), \ldots, u_{p}(t)$. However, on the other hand, choosing the values of $u_{1}(t), \ldots, u_{p}(t)$ too small can result in a significant reduction of the economic activity $E(t)$, which may result in bankruptcy of businesses and loss of livelihoods. Therefore, our goal in this section is to find optimal capacity control policy that maximize the economic activity under a constraint that the infection peak $I_{\text {peak }}$ remains bounded from above.

Suppose a finite time horizon $T$, a policy horizon $T_{u}=$ $T / q$ for $q \in \mathbb{N}$, an upper bound $\bar{I}>0$ on the infection peak, and all the parameters of the model (8) be given. Let $\mathbf{u} \in \mathcal{U}$, where $\mathcal{U}$ is the set of admissible capacity control policies $\mathbf{u}:[0, T] \rightarrow[0,1]^{p}$ such that, for every $t \in\left[(h-1) T_{u}, h T_{u}\right)$ and $h \in\{1, \ldots, q\}, \mathbf{u}(t)=\boldsymbol{\mu}_{h}$ for some $\boldsymbol{\mu}_{h}=\left[\begin{array}{lll}\mu_{1}^{h} & \cdots & \mu_{p}^{h}\end{array}\right]^{\mathrm{T}} \in[0,1]^{p}$. Then, the optimal capacity control policy is obtained by solving the following problem:

$$
\begin{aligned}
& \underset{\mathbf{u} \in \mathcal{U}}{\operatorname{maximize}} L(\mathbf{u}):=\frac{1}{T} \int_{0}^{T} \mathbf{e}^{\mathrm{T}} \mathbf{x}\left(t ; \mathbf{x}_{0}, \mathbf{u}\right) d t \\
& \text { subject to }\left\{\begin{array}{l}
\dot{\mathbf{x}}=\boldsymbol{\xi}(\mathbf{x})+\Phi(\mathbf{x}, \mathbf{u}) \mathbf{x} ; \mathbf{x}(0)=\mathbf{x}_{0} \\
I_{\text {peak }}(\mathbf{u}) \leq \bar{I}
\end{array}\right.
\end{aligned}
$$

where $I_{\text {peak }}(\mathbf{u})=\sup _{t \in[0, T]} \mathbf{g}^{\mathrm{T}} \mathbf{x}\left(t ; \mathbf{x}_{0}, \mathbf{u}\right)$ is given in (12) and the economic activity $E(t)=\mathbf{e}^{\mathrm{T}} \mathbf{x}(t)$ is given in 10 .

Example 3. Again, consider the example of Figure 1 with the mobility and epidemic parameters given in Table $\mathrm{I}$ and III respectively. Also, consider the parameters in Table III required by the optimal control problem (13). The optimal control problem is solved numerically for a time horizon $T=1680$ hours (i.e., 10 weeks) using a nonlinear programming solver fmincon in MATLAB with interior point algorithm. The solver returns a local minimum $\mathbf{u}_{\mathrm{opt}}(t)$ plotted in Figure 6 that satisfies the constraints (??) and is piecewise constant, where the policy horizon $T_{u}=336$ hours (i.e., 2 weeks). In particular, the constraint on the infection 


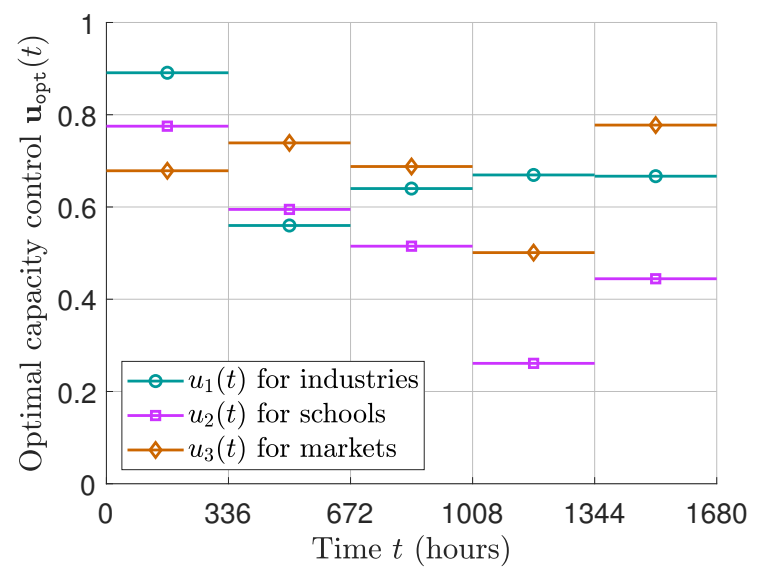

Fig. 6: Optimal capacity control policy $\mathbf{u}_{\text {opt }}$.

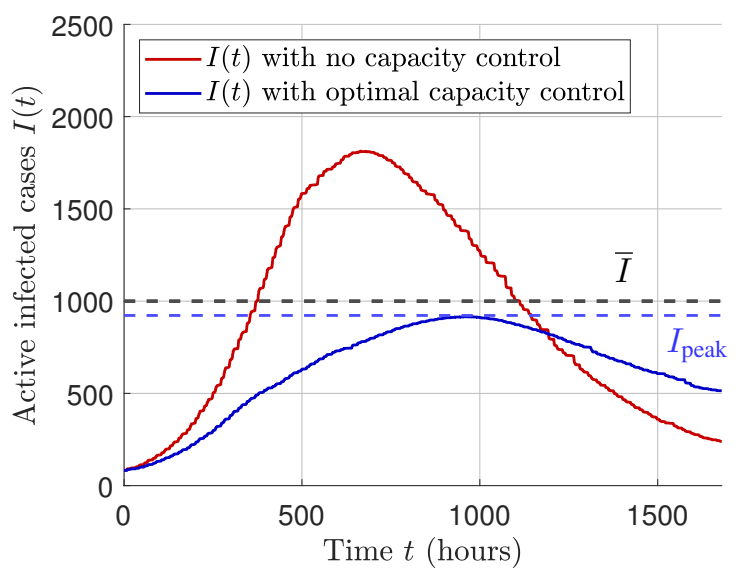

Fig. 7: Reduction of the number of infected cases $I(t)$ by controlling the operating capacities via optimal capacity control policy $\mathbf{u}_{\mathrm{opt}}$.

peak is satisfied and $I_{\text {peak }} \approx 922$ is less than $\bar{I}=1000$ as shown in Figure 7 Notice that in the beginning the optimal capacity control allows the operating capacities to be around $70-90 \%$ of the nominal capacities of destinations. However, as the number of infected cases increase, the value of the optimal capacity control decreases for the next four steps until $8 T_{u}=1344$ hours (i.e., 8 weeks) to mitigate the infection spread. Then, in the last interval $\left[8 T_{u}, T\right]$, the optimal capacity control increases to allow more people visiting the destinations because the infected cases have started to decrease. In Figure 8, we plot the economic activity for three cases: (i) when no capacity control policy is implemented, (ii) when the capacity control policy limits the operating capacities to $50 \%$ of the nominal capacities of destinations, and (iii) when the optimal capacity control policy $\mathbf{u}_{\text {opt }}(t)$ shown in Figure 6 is applied. Notice that the optimal capacity control $\mathbf{u}_{\mathrm{opt}}(t)$ increases the economic activity as compared to the cases when $\mathbf{u}(t)=0.51_{3}$ while keeping the $I_{\text {peak }}$ under the bound $\bar{I}$.

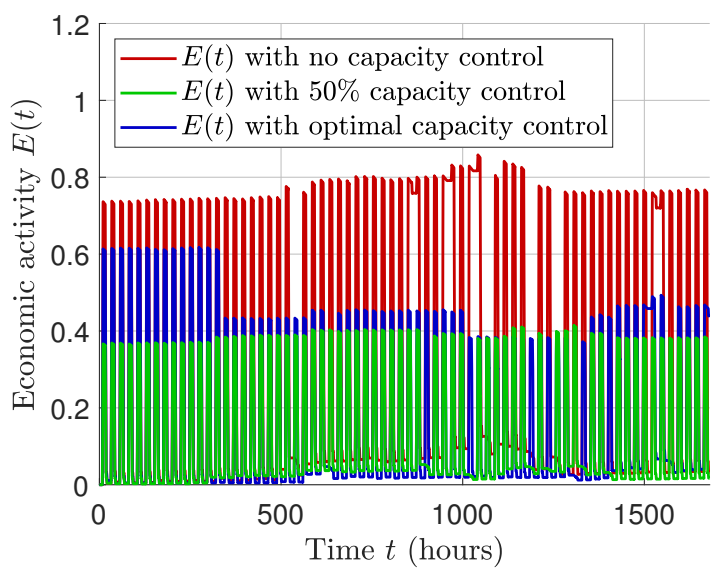

Fig. 8: Economic activity (i) without capacity control policy, i.e., $\mathbf{u}=\mathbf{1}_{3}$, (ii) with $50 \%$ capacity control policy $\mathbf{u}=0.5 \mathbf{1}_{3}$, and (iii) with optimal capacity control policy $\mathbf{u}_{\mathrm{opt}}$.

\section{Optimal Schedule Control Policy}

We formulate an epidemic mitigation policy that alters the destination schedules and mobility windows. That is, for every $j \in \mathcal{D}_{k}$, the destination schedule is altered as

$$
\mathbb{S}_{j}=\left[a_{j}, \min \left(s_{k}, b_{j}\right)\right)
$$

where $s_{k} \in[\underline{s}, 24)$ is the schedule control that enforces that all destinations of category $k$, for $k=1, \ldots, p$, must be closed after $s_{k}$ hour, respectively, and $\underline{s} \geq 0$ is the lower bound on $s_{k}$. Such a policy limits the spread of infection by reducing the daily amount of time people spend at destinations. It also alters the mobility windows

$$
\begin{aligned}
\mathbb{D}_{i j} & =\left[t_{i j}, \min \left(t_{i j}+\tau_{i j}, s_{k}\right)\right) \\
\mathbb{D}_{j i} & =\left[\min \left(t_{j i}, s_{k}\right), \min \left(t_{j i}, s_{k}\right)+\tau_{j i}\right) .
\end{aligned}
$$

for $i \in \mathcal{V}_{o}$ and $j \in \mathcal{D}_{k}$. That is, people cannot go from $i$ to $j$ after $s_{k}$ hour and people at $j$ must return to $i$ after $s_{k}$ hour.

Let $\mathbf{s}=\left[\begin{array}{lll}s_{1} & \cdots & s_{p}\end{array}\right]^{\mathrm{T}}$ be the schedule control policy of all destination categories. Then, the problem is to find an optimal $\mathbf{s}$ such that the economic activity is maximized while keeping the infection peak bounded by $\bar{I}$. The schedule control policy complements the capacity control policy obtained by solving (13) when there are lower bounds on the capacity control policy. These lower bounds correspond to minimum operating capacities of certain destinations that are required for functioning of the society. This is because some destinations, like hospitals and markets, are essential and their operating capacities cannot be reduced beyond a minimum bound. In other words, for all $k \in\{1, \ldots, p\}$ and $h \in\{1, \ldots, q\}$, we assume constant capacity control policy $\mathbf{u}(t)=\boldsymbol{\mu}$, for all $t \in[0, T]$, where $\boldsymbol{\mu} \in[0,1]^{p}$ states the minimum allowed capacity control policy of $p$ destination categories. In the presence of these lower bounds, the problem (13) may become infeasible and the infection peak may no longer be bounded. Thus, implementation of optimal schedule control policy $\mathbf{s}$ may help in containing 


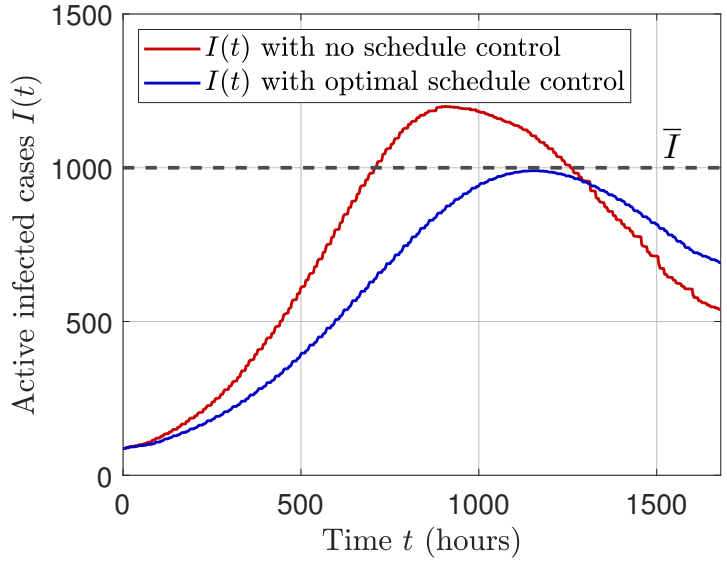

Fig. 9: Reduction of the number of active infected cases $I(t)$ by controlling destination schedules and mobility windows via optimal schedule control policy $\mathbf{s}_{\mathrm{opt}}$.

the infections while also allowing economic activity at destinations.

Suppose a finite time horizon $T$, an upper bound $\bar{I}>0$ on the infection peak, a constant capacity control policy $\mathbf{u}(t)=$ $\boldsymbol{\mu} \in[0,1]^{p}, \forall t \in[0, T]$, and the parameters of the model (8) are given. Then, the optimal schedule control policy is obtained by solving the following problem:

$$
\begin{aligned}
& \underset{\mathbf{s} \in[\underline{s}, 24)^{p}}{\operatorname{maximize}} L(\mathbf{s}):=\frac{1}{T} \int_{0}^{T} \mathbf{e}^{\mathrm{T}} \mathbf{x}\left(t ; \mathbf{x}_{0}, \mathbf{s}\right) d t \\
& \text { subject to }\left\{\begin{array}{l}
\dot{\mathbf{x}}=\boldsymbol{\xi}(\mathbf{x})+\Phi(\mathbf{x}, \mathbf{s}) \mathbf{x} ; \mathbf{x}(0)=\mathbf{x}_{0} \\
I_{\text {peak }}(\mathbf{s}) \leq \bar{I}
\end{array}\right.
\end{aligned}
$$

where $I_{\text {peak }}(\mathbf{s})=\sup _{t \in[0, T]} \mathbf{g}^{\mathrm{T}} \mathbf{x}\left(t ; \mathbf{x}_{0}, \mathbf{s}\right)$ is given in 12$]$ and the economic activity $E(t)=\mathbf{e}^{\mathrm{T}} \mathbf{x}(t)$ given in 10 .

Example 4. Consider again the example of Figure 1 and the parameters given in Table II, II, and III where we change the policy horizon $T_{u}=T$ since the schedule control policy $\mathbf{s}$ is constant throughout $[0, T]$. Let the capacity control policy $\mathbf{u}(t)=\left[\begin{array}{lll}0.75 & 0.75 & 0.75\end{array}\right]^{\mathrm{T}}, \forall t \in$ $[0, T]$, i.e., the operating capacities of destinations should be $75 \%$ of their nominal capacities. Then, solving (14) numerically by using fmincon solver in MATLAB with interior point algorithm, we obtain a local minimum $\mathbf{s}_{\mathrm{opt}}=$ $\left[\begin{array}{lll}14.54 & 13.75 & 16.10\end{array}\right]^{\mathrm{T}}$, which means that the industries must be closed after 14.54 hour $(02: 32 \mathrm{pm})$, the schools must be closed after 13.75 hour $(01: 45 \mathrm{pm})$, and the markets must be closed after 16.10 hour (04:06 pm). Figure 9 shows that the constraint on the infection peak is satisfied.

\section{Conclusion}

We developed an urban human mobility model on network of origins and destinations that incorporates the process of epidemic spread at each location. The model is described by the flows that transfer people from origins to destinations and back to origins every day. The flows capture the daily patterns of mobility in an urban environment through the gating functions that depend on the destination schedules and mobility windows. At each location, the disease spreads through the interaction of susceptible and infected people, where the infection rate depends on the number of people in that location. We study two optimal control policies, capacity control and schedule control, that mitigate the epidemic spread while maximizing the economic activity at each destination. The optimal capacity control policy maximizes the economic activity by allowing maximum allowable number of people at each destination under the constraint that the infection peak remains bounded. The optimal schedule control policy maximizes the economic activity by allowing maximum allowable time that people spend daily at destinations under the constraint that the infection peak remains bounded.

The future investigations include the model predictive control formulation of optimal control problems. A work in progress is the validation of model with the urban mobility data and regional epidemic statistics and the development of web interface to illustrate our proof of concept.

\section{REFERENCES}

[1] L. Sattenspiel and K. Dietz, "A structured epidemic model incorporating geographic mobility among regions," Mathematical biosciences, vol. 128 , no. 1-2, pp. 71-91, 1995.

[2] J. Arino and P. Van den Driessche, "A multi-city epidemic model," Mathematical Population Studies, vol. 10, no. 3, pp. 175-193, 2003.

[3] D. Balcan, B. Gonçalves, H. Hu, J. J. Ramasco, V. Colizza, and A. Vespignani, "Modeling the spatial spread of infectious diseases: The GLobal Epidemic and Mobility computational model," Journal of computational science, vol. 1, no. 3, pp. 132-145, 2010.

[4] C. Poletto, M. Tizzoni, and V. Colizza, "Human mobility and time spent at destination: Impact on spatial epidemic spreading," Journal of theoretical biology, vol. 338, pp. 41-58, 2013.

[5] E. Frias-Martinez, G. Williamson, and V. Frias-Martinez, "An agentbased model of epidemic spread using human mobility and social network information," in 2011 IEEE 3rd International Conference on Privacy, Security, Risk and Trust and 2011 IEEE 3rd International Conference on Social Computing, 2011, pp. 57-64.

[6] L. Pappalardo, F. Simini, S. Rinzivillo, D. Pedreschi, F. Giannotti, and A.-L. Barabási, "Returners and explorers dichotomy in human mobility," Nature communications, vol. 6, no. 1, pp. 1-8, 2015.

[7] R. Pastor-Satorras, C. Castellano, P. Van Mieghem, and A. Vespignani, "Epidemic processes in complex networks," Reviews of modern physics, vol. 87, no. 3, p. 925, 2015.

[8] M. Nadini, L. Zino, A. Rizzo, and M. Porfiri, "A multi-agent model to study epidemic spreading and vaccination strategies in an urban-like environment," Applied Network Science, vol. 5, no. 1, pp. 1-30, 2020.

[9] S. Song, Z. Zong, Y. Li, X. Liu, and Y. Yu, "Reinforced epidemic control: Saving both lives and economy," arXiv preprint arXiv:2008.01257, 2020.

[10] H. Hethcote, "The mathematics of infectious diseases," SIAM Review, vol. 42 , no. 1 , pp. 599-653, 2000. 